\title{
Polylysine-Coated Diamond Nanocrystals for MALDI-TOF Mass Analysis of DNA Oligonucleotides
}

\section{L. Kong, L. C. L. Huang, S.-C. V. Liau, C.-C. Han, and H.-C. Chang}

DNA adsorption. MALDI-TOF MS was used to characterize DNA adsorption by analyzing the oligonucleotides in both precipitate and supernatant after the diamond nanocrystal pretreatment. With PL-coated diamonds as the SPE supports, the majority of the DNA oligonucleotides are detected in the precipitate (Figs. S1a and S1b), whereas most of the oligonucleotides remain in the supernatant after the pretreatment with diamonds without PL coating (Figs. S1c and S1d). The results reflect the high affinity of PL-coated diamonds for DNA oligonucleotides.
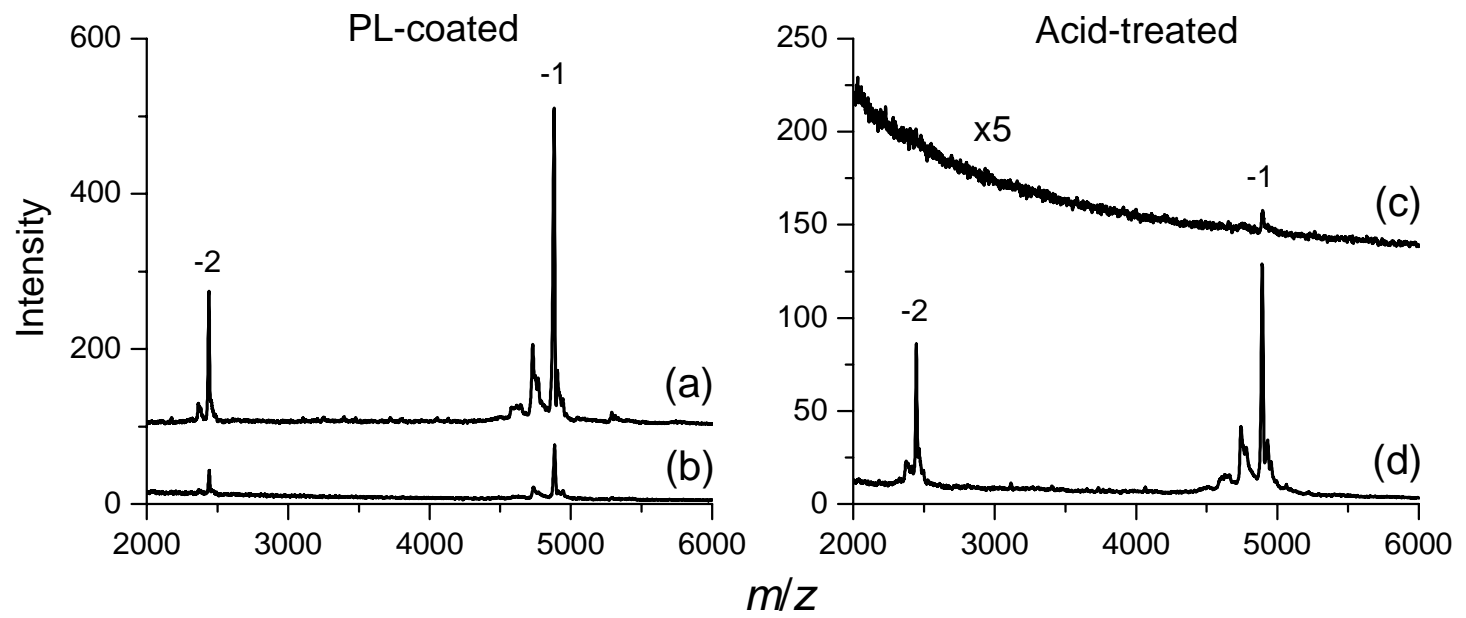

Figure S1. MALDI-TOF mass spectra of d(ATCGGCTAATCGGCTA), obtained by analysis of $(\mathrm{a}, \mathrm{c})$ precipitate and (b,d) supernatant after pretreatment of the sample solutions with PL-coated and acidtreated diamond nanocrystals, respectively. Curves (a) and (c) are shifted vertically for clarity. 


\section{Supporting information}

DNA enrichment. Sample enrichment by use of PL-coated diamond nanocrystals was observed for a variety of DNA oligonucleotides, including d(ATCGGCTAATCGGCTA) (a 16-mer, mass 4881), $\mathrm{dT}_{16}$ (mass 4805), $\mathrm{dC}_{16}$ (mass 4565), $\mathrm{dA}_{16}$ (mass 4949), lambda gt11 (a 24-mer, mass 7396) and biotinylated T7 primer (a 24-mer, mass 8055). Although the degree of the enrichment varies with the sample composition, it is always greater than a factor of 10 (Figs. S2 and S3).
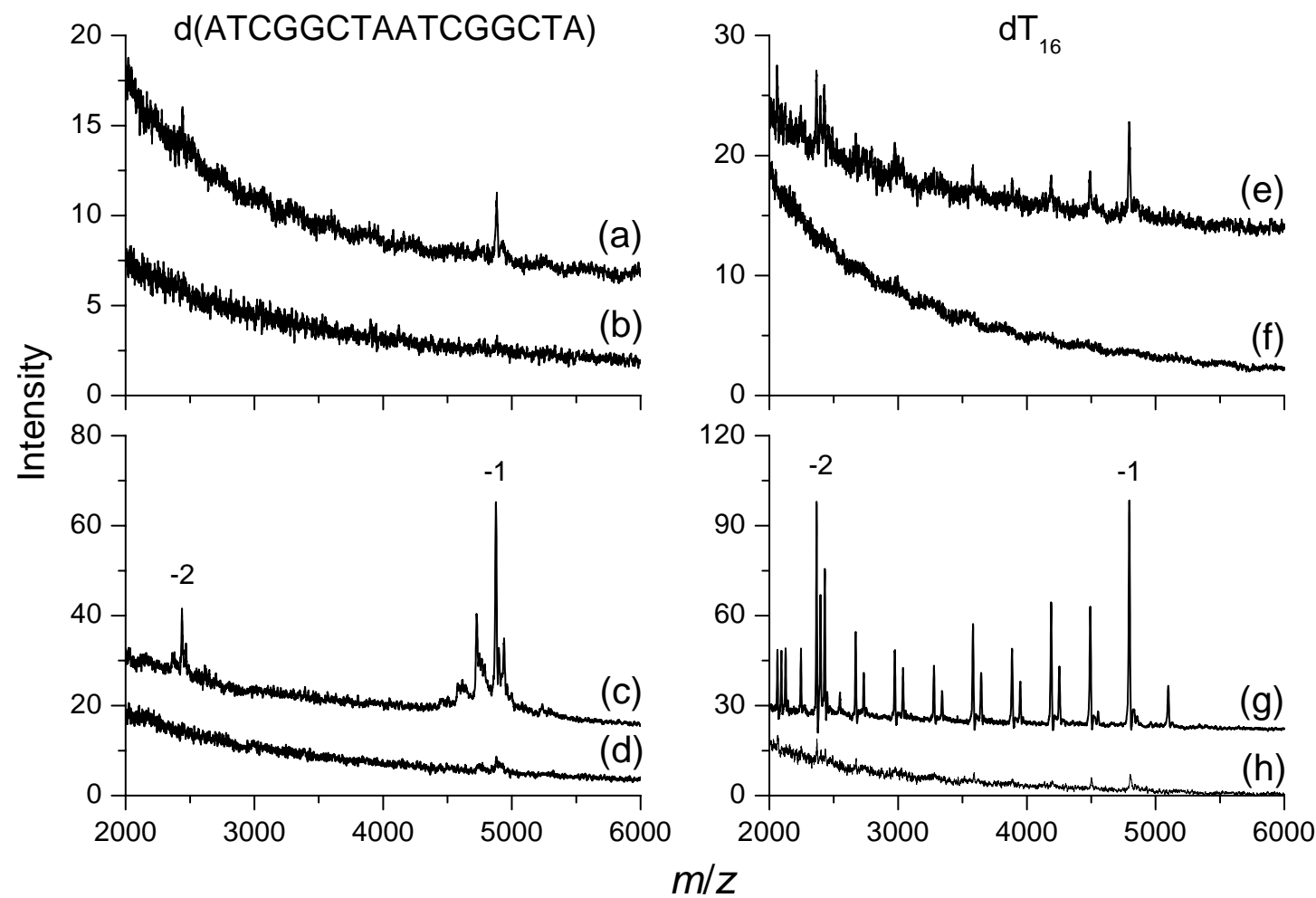

Figure S2. MALDI-TOF mass spectra of d(ATCGGCTAATCGGCTA) and $\mathrm{dT}_{16}$ obtained by direct analysis of $1 \mu \mathrm{L}$ solutions at a concentration of (a,e) $10 \mathrm{nM}$ and (b,f) $5 \mathrm{nM}$, respectively. With the PLcoated diamond pretreatment for $500 \mu \mathrm{L}$ solutions, the concentration can be readily lowered to (c,g) 5 nM and (d,h) 100 pM. Curves (a), (c), (e) and (g) are shifted vertically for clarity. The equally spaced doublets appearing regularly in (g) at $m / z<4600$ correspond to DNA fragment ions. 


\section{Supporting information}

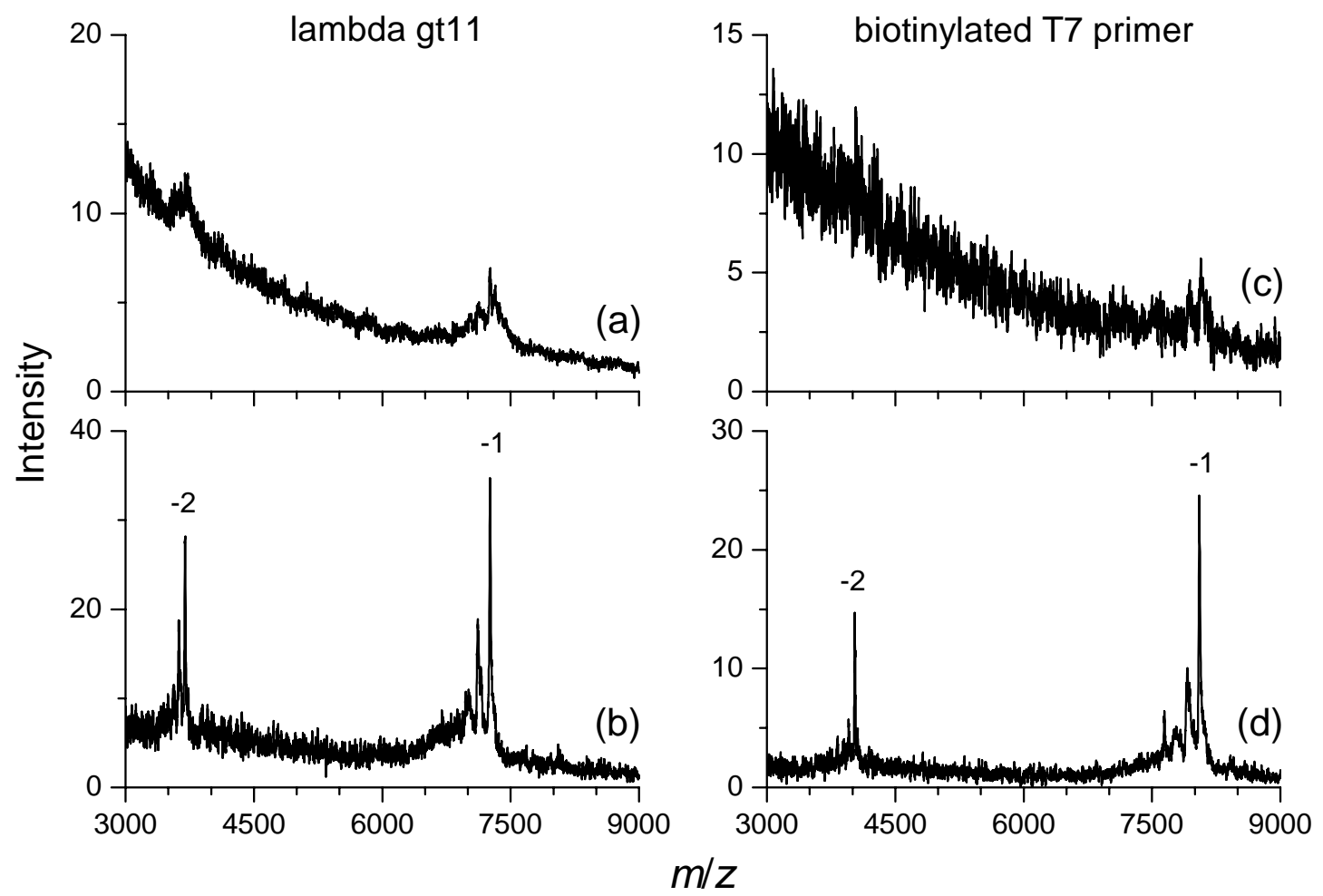

Figure S3. MALDI-TOF mass spectra of lambda gt11 [d(GGTGGCGACGACTCCTGGAGCCCG)] and biotinylated T7 primer [5'-biotin-d(CTCGAGTAATACGACTCACTCTATAGG)-3’], obtained by (a,c) direct analysis of $200 \mathrm{nM}$ solution $(1 \mu \mathrm{L})$ and (b,d) analysis of $100 \mathrm{nM}$ solution $(500 \mu \mathrm{L})$ preconcentrated with PL-coated diamonds, respectively. 